\title{
Vite svuotate. Per una critica dell'impatto psicosociale del capitalismo contemporaneo
}

Marco Solinas*

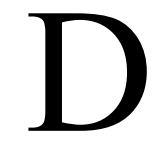
alle ricerche sociali ed epidemiologiche realizzate soprattutto nel corso dell'ultimo decennio è emerso che nei paesi occidentali, ad iniziare all'incirca dai primi anni settanta, il malessere psichico ha virato progressivamente verso un quadro segnato da una annichilente sensazione di vuoto interiore e di impotenza. L'insensatezza, la stanchezza, la passività registrate sono state ricondotte entro il quadro della depressione, categoria nosologica che è andata nel frattempo costantemente ampliandosi; disturbo di cui è stata infine decretata la diffusione epidemica, non solo in senso strettamente clinico. Nello stesso periodo e nella stessa area, si è andato imponendo il nuovo modello neoliberista di accumulazione capitalistica, caratterizzato tra gli altri elementi dalla flessibilizzazione del mercato del lavoro e dallo smantellamento del welfare state; due fattori che hanno alterato in profondità gli equilibri sociali complessivi e le condizioni di vita dei lavoratori. Il nuovo modello ha comportato sincronicamente delle trasformazioni altrettanto significative del quadro etico, morale e normativo, e più in generale culturale e politico delle società occidentali, radicalizzando ed esasperando tendenze di lungo corso. È tra questi due poli, l'affermazione del capitalismo neoliberista e il vuoto psichico, che traccerò dei ponti. La tesi di fondo che proverò qui a delucidare per sommi capi è che l'incremento delle derive di natura depressiva abbia come concausa l'impatto del nuovo modello capitalistico. Cercherò quindi di individuare dei nessi causali tra queste due dimensioni, con la speranza di contribuire a far luce sull'impatto psicosociale del capitalismo contemporaneo e di approntare, nel contempo, degli strumenti concettuali atti a delinearne una critica. 
Sul piano degli strumenti analitici e teorici adottabili, la realizzazione di un tale obiettivo trova un primo retroterra di orientamento empirico nelle ormai classiche analisi delle «corrosive» conseguenze del nuovo capitalismo sul carattere e sullo stile di vita dei lavoratori flessibili proposte da Richard Sennett; contributi nei quali, di tanto di tanto, emergono anche dei richiami ai fenomeni di tipo depressivo ${ }^{1}$. Se mi muoverò in questa direzione cercherò però, oltre a focalizzare meglio la questione del vuoto, di ricondurla entro il framework teoretico delineato da Axel Honneth. Trovo infatti del tutto convincente la sua lettura, rielaborata direttamente sulla scia del capolavoro di Boltanski e Chiapello ${ }^{2}$, delle trasformazioni del quadro normativo contemporaneo nei termini di un rovesciamento paradossale delle istanze etiche e morali originariamente emancipatorie avanzate dai movimenti di protesta degli anni sessanta ${ }^{3}$. A mio avviso, una tale impostazione conduce però alla necessità di delineare un'interpretazione di taglio socioculturale dell'epidemia depressiva differente rispetto a quella oggi prevalente, canonizzata da Alain Ehrenberg nella sua brillante ricostruzione storico-psichiatrica del fenomeno ${ }^{4}$. Questa revisione delle correlazioni tra la forma e le funzioni del nuovo quadro normativo approntato dal capitalismo contemporaneo e la diffusione di massa dei sintomi depressivi, credo conduca a sua volta alla necessità di riflettere sulla dimensione etico-politica dei processi complessivi in gioco. Ampliamento prospettico che trova nel lascito di Antonio Gramsci strumenti, temi e questioni preziosi, a iniziare dal concetto di egemonia ${ }^{5}$. Ed è richiamandone la tematizzazione che cercherò di ricostruire, all'interno del framework teoretico-riconoscitivo deli-

1. Mi riferisco soprattutto a R. Sennett, The Corrosion of Character. The Personal Consequences of Work in the New Capitalism, New York-London, 1999, trad. it. L'uomo flessibile. Le conseguenze del nuovo capitalismo sulla vita personale, Feltrinelli, Milano 1999 e Id., The Culture of New Capitalism, New Haven-London 2006, trad. it. La cultura del nuovo capitalismo, il Mulino, Bologna, 2006.

2. L. Boltanski, E. Chiapello, Le nouvel esprit du capitalisme, Gallimard, Paris 1999.

3. Da questa prospettiva riprenderò soprattutto A. Honneth, Organisierte Selbstverwirklichung. Paradoxien der Individualisierung, Frankfurt/New York 2002, trad. it. Autorealizzazione organizzata. Paradossi dell'individualizzazione, in Id., Capitalismo e riconoscimento, Firenze University Press, Firenze, 2010; e M. Hartmann, A. Honneth, Paradoxien des Kapitalismus. Ein Untersuchungsprogramm, «Berliner Debatte Initial», 15/1 (2004), pp. 4-17, trad. it. Paradossi del capitalismo. Un programma di ricerca, in Id., Capitalismo e riconoscimento, cit.

4. Cfr. A. Ehrenberg, La fatigue d'être soi. Dépression et société, Paris 1998, trad. it. La fatica di essere se stessi. Depressione e società, Einaudi, Torino 1999.

5. Mi riferisco soprattutto a A. Gramsci, Quaderni del carcere, Quaderno 11 (XVIII) "/ntroduzione alla filosofia”, § 12, e Quaderno 13 (XXX) “Noterelle sul Machiavelli”, § 14-17. 
neato da Honneth ${ }^{6}$, le dinamiche circolari tra le reazioni depressive e l'inibizione della nascita di lotte sociali di natura emancipativa. L'analisi dell'impatto psicosociale del capitalismo contemporaneo, declinato dalla prospettiva negativa delle reazioni di massa interpretabili come regressive, verrà così a rappresentare il fulcro di una proposta volta ad ampliare il compito fondamentale di una rinnovata teoria critica: lavorare riflessivamente sui potenziali emancipativi frustrati della sofferenza sociale di tipo depressivo e regressivo.

Jel quadro epidemiologico dei disagi, dei disturbi e delle psicopatolo1 gie che affliggono le società occidentali, negli ultimi quattro decenni è stato registrato un incremento dei sintomi depressivi senza precedenti. Il vuoto, o se si vuole l'insensatezza, l'affaticamento, il disorientamento di matrice depressiva, sono così ampiamente straripati dalla cornice psichiatrica e psicoanalitica per imporsi prepotentemente all'attenzione dei ricercatori sociali. Tra i vari studi dedicati alla questione, spicca quello accuratissimo di Ehrenberg, edito nel 1998 e presto divenuto un riferimento fondamentale. Al di là delle ricche analisi storico-ricostruttive e descrittive, viene qui avanzata la tesi secondo cui lo straordinario aumento dei sintomi depressivi sarebbe direttamente correlato alla maggiore «autonomia» e «libertà» conquistata dai cittadini occidentali soprattutto a partire dagli anni sessanta: «il depresso» non rappresenterebbe che «il rovescio negativo» di tale ampliamento, in quanto soggetto che non riesce a farsi carico della «responsabilità» di cui ora può godere ${ }^{7}$. In breve, i cittadini e i lavoratori occidentali sarebbero (finalmente) condannati ad una libertà di ascendenza nietzschiana di cui (purtroppo) non riuscirebbero a sostenere il peso, non ne sarebbero «all'altezza». E così, affaticati da un tale compito, impostogli dal

6. Riprenderò soprattutto a A. Honneth, Kampf um Anerkennung. Grammatik sozialer Konflikte, Frankfurt/Main 1992, trad. it. Lotta per il riconoscimento. Proposte per un'etica del conflitto, il Saggiatore, Milano, 2002 e N. Fraser, A. Honneth, Umverteilung oder Anerkennung? Eine politisch-philosophische Kontroverse, Suhrkamp, Frankfurt/Main, 2003 - Idd., Redistribution or Recognition?: A Political-Philosophical Exchange, Verso, London, 2003 trad. it. Redistribuzione o riconoscimento? Una controversia politico-filosofica, Meltemi, Roma, 2007.

7. Cfr. A. Ehrenberg, La fatigue d'être soi. Dépression et société, Paris 1998, trad. it. La fatica di essere se stessi. Depressione e società, Einaudi, Torino 1999, soprattutto pp. $222-$ $223,254-256,7-10,300-301$. 
nuovo quadro etico, economico e normativo, troverebbero uno scomodo rifugio nella malattia. È così che l'antica e nobile melanconia si sarebbe trasformata in una nefasta patologia di massa.

Ora, è indubitabile che in molti ambiti della vita sociale, nel corso degli ultimi tre o quattro decenni, vi sia stato un rapido e radicale ampliamento degli spazi dell'autonomia e della libertà individuale, ad esempio in quello delle relazioni affettive, e più in generale un'accelerazione del processo di individualizzazione. Dinamiche che certamente possono aver contribuito a disorientare, generando una condizione complessiva di incertezza, insicurezza, stanchezza, che forse potremmo interpretare anche nei termini di anomia. Tuttavia, allorché ci si sposta sul piano del lavoro, quindi di quelle condizioni fondamentali di vita che dettano i ritmi della quotidianità, le disponibilità finanziarie, e influenzano in modo determinante gli spazi e i tempi delle stesse relazioni affettive, il quadro cambia radicalmente. È divenuto infatti sempre più chiaro che uno dei meccanismi fondamentali introdotti dal capitalismo neoliberista, la flessibilizzazione del mercato (e delle forme) del lavoro, non ha aumentato, ma ha invero ristretto radicalmente gli spazi dell'autonomia e della libertà dei lavoratori, privandoli di diritti fondamentali per assoggettarli a forme di subordinazione e sfruttamento, talvolta quanto mai brutali, che lunghe lotte politiche e sociali erano riuscite a bandire, soprattutto dai paesi europei ${ }^{8}$. Masse in costante crescita di lavoratori flessibili, frammentati, atomizzati e de-sindacalizzati com'erano stati soltanto in un passato remoto, si sono così ritrovati nuovamente in balia di un mercato reso ancor più selvaggio dal concomitante smantellamento del welfare.

Privati delle garanzie sul futuro precedentemente assicurate dallo stato sociale, assillati dalla necessità di rinnovare ininterrottamente contratti a breve termine, impauriti dallo spettro delle continue ristrutturazioni aziendali, i nuovi lavoratori sono stati costretti a rimodulare la propria esistenza sul breve termine. L'incertezza, l'insicurezza, la stanchezza che emerge nel fenomeno depressivo trova qui una delle sue radici materiali. Ed è infatti su questa linea che soprattutto nel corso dell'ultimo decennio sono emersi diversi studi volti a mostrare la diretta correlazione tra la flessibilizzazione del mercato del lavoro, che si traduce puntualmente in una precarizzazione delle condizioni di vita, e la

8. Vedi la magistrale analisi di L. Gallino, // lavoro non è una merce. Contro la flessibilità, Laterza, Roma-Bari, 2007, soprattutto pp. $57 \mathrm{sgg}$., $75 \mathrm{sgg}$. 
crescita dei fenomeni depressivi ${ }^{9}$. Lo stesso Ehrenberg, nonostante la sua tesi di fondo, fin dal 1998 non poté del resto non riscontrare che sul piano più strettamente descrittivo non soltanto veniva confermata la classica correlazione tra scarsi salari, disoccupazione e malessere psichico, ma più precisamente tra l'epidemia depressiva e la sempre maggior «precarietà» del lavoro indotta dal nuovo modello economico ${ }^{10}$.

Ton appena ci si sposta sul piano simbolico, la questione diviene però infinitamente più complessa. Anzitutto perché la flessibilità, che rappresenta uno dei pilastri fondativi della nuova forma di accumulazione capitalistica in quanto meccanismo che permette la drastica riduzione del costo del lavoro e del rischio di impresa ${ }^{11}$, è stata ed è oggetto di continui tentativi di giustificazione e legittimazione concernenti una molteplicità di piani differenti. Su quello etico e morale, è stata presentata come uno dei più preziosi dispositivi atti a incrementare ulteriormente l'autonomia, la libertà di scelta individuale, l'iniziativa personale e il merito; quindi atta a realizzare quei principi basilari a cui il movimento neoliberista non si stanca di appellarsi. Al riguardo, la tesi che trovo più convincente è stata delineata da Honneth riprendendo il capolavoro di Boltanski e Chiapel$10^{12}$. Si tratta del fatto che il capitalismo neoliberista ha sostanzialmente inglobato, e quindi rovesciato le istanze emancipatorie avanzate dai movimenti di protesta degli anni sessanta, piegandole alla logica dell'accumulazione; dinamica che presenta così un andamento eminentemente paradossale ${ }^{13}$. Più da vicino, Honneth ha richiamato l'attenzione

9. Cfr. p. es. L. Artazcoz, J. Benach, C. Borrell, I. Cortès, Social inequalities in the impact of flexible employment on different domains of psychosocial health, in «Journal of Epidemiology and Community Health», 59 (2005), pp. 761-767; Ch. Dejours, Aliénation et clinique du travail, in Nouvelles aliénations, in «Actuel Marx», n. 39 (2006), pp. 123-144: 125-135.

10. Vedi A. Ehrenberg, La fatica di essere se stessi, cit., pp. 251-157.

11. Cfr. L. Gallino, I/ lavoro non è una merce. Contro la flessibilità, Laterza, Roma-Bari 2007, cit., pp. 27 sgg.

12. Cfr. L. Boltanski, E. Chiapello, Le nouve/ esprit du capitalisme, cit., soprattutto pp. $501 \mathrm{sgg}$. 13.Cfr. M. Hartmann, A. Honneth, Paradoxien des Kapitalismus. Ein Untersuchungsprogramm, «Berliner Debatte Initial», 15/1 (2004), pp. 4-17, trad. it. Paradossi del capitalismo. Un programma di ricerca, in Id., Capitalismo e riconoscimento, Firenze University Press, Firenze 2010; Id., Organisierte Selbstverwirklichung. Paradoxien der Individualisierung, Frankfurt/New York 2002, trad. it. Autorealizzazione organizzata. Paradossi dell'individualizzazione, in Id., Capitalismo e riconoscimento, cit. 
sulla funzione di due istanze particolarmente significative: la responsabilità personale e l'autorealizzazione.

A quest'ultimo ideale, nell'accezione di una soggettività aperta e sperimentale, è stato ricondotto il modello contemporaneo del sé flessibile: tale da poter cogliere le sfuggenti opportunità di un mercato del lavoro sempre più deregolato come se si trattasse, sempre o per lo più, di imperdibili chance atte a favorire un ininterrotto percorso di crescita individuale ed esistenziale dei lavoratori. L'ideale dell'autorealizzazione si è così trasformato in un «modello di aspettative istituzionalizzato», ormai sostanzialmente «esterno» a quelle esigenze e a quei desideri dai quali era nato, al quale i soggetti devono ora conformarsi, come se rispecchiasse e veicolasse ancora quegli stessi valori che ha invero perduto ${ }^{14}$. $\mathrm{E}$ così, appellandosi al principio intrinsecamente anti-disciplinare della libera espressione e dell'autorealizzazione, i lavoratori vengono di fatto assoggettati al nuovo disciplinamento di un sé flessibilizzato da meccanismi alla cui morsa è sempre più difficile sottrarsi. Anche la funzione dell'ideale della responsabilità personale, di cui nel contempo viene denunciato il progressivo e costante indebolimento, sembra essersi rovesciata: mentre fino agli anni Sessanta era stato rivendicato in contrapposizione agli alienanti e spersonalizzanti meccanismi di disciplinamento di matrice fordista-taylorista, e possedeva quindi un contenuto emancipatorio, oggi pare contribuire soprattutto ad addossare agli individui, in negativo, la responsabilità per le proprie condizioni di vita, contribuendo così ad arricchire l'arsenale degli strumenti adottati per giustificare la destrutturazione del welfare. La dinamica, paradossale, conduce qui a un'impasse: i soggetti vengono indotti a farsi carico, individualmente, della responsabilità di condizioni che rimandano a delle precondizioni di cui essi non possono certo essere gli unici responsabili, giustificando nel contempo i meccanismi volti a decostruire taluni dei presupposti socioeconomici fondamentali necessari a rafforzare la possibilità stessa di tale assunzione. Convergendo in un movimento a spirale, gli appelli alla responsabilità personale e all'autorealizzazione sono andati così a formare il nucleo legittimatorio etico e morale del quadro normativo individualistico e libertario neoliberista, fornendo i principi basilari atti a giustificare le nuove forme di sfruttamento approntate attraverso la flessibilizzazione del mercato del lavoro, nonché il sincronico smantellamento del welfare. Si tratta, dunque, di una configurazione ideologica nel senso più classico: maschera i propri reali intenti appellandosi a quegli stessi principi che di fatto nega ${ }^{15}$.

14. Così A. Honneth, Autorealizzazione organizzata, cit., p. 43.

15. Per una magistrale e aggiornata ricostruzione dell' ideologia in questo senso vedi Jaeggi

R., Per una critica dell'ideologia, in «Iride», 55, XXI (2008), pp. 595-616. 
$U^{n}$ 'emblematica esemplificazione empirica dell'impatto depressivo innescato dall'esasperazione del ruolo conferito alla responsabilità personale nel nuovo quadro normativo credo sia offerta dall'analisi delineata da Sennett delle letture e delle reazioni emotive - nel doppio piano circolare dell'auto percezione soggettiva e della canonizzazione e talvolta stigmatizzazione sociale - suscitate in un gruppo di lavoratori statunitensi dalle esperienze di perdita del lavoro, nella fattispecie in casi dovuti a processi di ristrutturazione aziendale ${ }^{16}$. In una prima forma paradigmatica l'esperienza viene interpretata nei termini di un personal failure: i soggetti si attribuiscono la responsabilità personale di un qualche errore di valutazione, ritenendo così di non essere stati all'altezza di quelle che interpretano in certo qual modo come sfide lanciate loro dalla realtà sociale. Lettura che induce l'emersione di sentimenti di vergogna, colpa e inadeguatezza tali da condurre a un ripiegamento su se stessi e a un generale ritiro dalla vita sociale di matrice in senso lato depressiva. In una seconda forma, altrettanto paradigmatica, che a me pare complementare alla prima, l'esperienza viene interpretata come un evento rispetto al quale il soggetto coinvolto si ritiene, ed è perlopiù considerato tale anche socialmente, come una «vittima predestinata»: oggetto di una ingiustizia perversa riconducibile a dinamiche del tutto al di fuori del suo controllo, di cui perciò non ritiene di essere in alcun modo responsabile. Interpretazione che genera sentimenti ed emozioni di sconforto, rancore e rabbia impotente, anch'essi chiaramente riconducibili al paradigma depressivo.

Lasciando ora sullo sfondo la questione complessiva del ruolo esercitato dal nuovo quadro culturale nell'edificazione di un sé che oscilla tra una condizione di onnipotenza e una di impotenza, e che lo espone anche da questa prospettiva a derive bipolari, ciò che vorrei sottolineare di queste due reazioni complementari è il grado e la qualità della dinamica di psicologizzazione e di personalizzazione della realtà sociale che entrambe, in modo analogo, sottendono e avallano. Il singolo individuo si percepisce, ed è perlopiù considerato socialmente, quale unico responsabile, oppure quale vittima predestinata, quindi del tutto non responsabile, di quelli che vengono interpretati come insuccessi (o successi) personali. Un caso come questo, che derubricherei alla voce «americanismo e flessibilismo», viene dun-

16. Cfr. Sennett R., L'uomo flessibile, cit., capitolo 7; e Id., La cultura del nuovo capitalismo, cit., pp. 79 sgg. 
que a rappresentare una stilizzazione empirica dell'impatto psicosociale esercitato dalla «cultura del nuovo capitalismo»: le tendenze spiccatamente individualistiche del liberismo classico sono state portate all'estremo e, dal mondo anglosassone, sono approdate ormai da tempo in Europa.

\section{IV}

Dosto che il nuovo quadro etico e morale coopera in modo determinante (sul piano simbolico) alla diffusione di massa di reazioni di natura depressiva, credo si ponga con forza la necessità di approntare degli strumenti analitici e teoretici in grado di contribuire riflessivamente al rovesciamento di tale dinamica. Da questa prospettiva, il compito di sviluppare una critica dell'impatto psicosociale del capitalismo contemporaneo deve avere come fine prioritario la riattivazione di quei potenziali emancipativi immanenti alla sofferenza sociale che vengono frustrati in tali reazioni, e quindi il dirottamento liberatorio delle cariche emotive e pulsionali in gioco. Su questo piano, il framework della nuova teoria critica elaborata da Axel Honneth rappresenta un ottimo ausilio, soprattutto perché insiste, a monte, sulla sofferenza psicologico-morale provata dai soggetti allorché subiscono delle esperienze di ingiustizia che egli interpreta alla luce della categoria del disrispetto (Missachtung), concernenti nella fattispecie il piano del diritto e della stima sociale: è questa la molla che innesca le dinamiche delle lotte sociali ${ }^{17}$, fornendone la base motivazionale ${ }^{18}$. Poiché le reazioni di natura depressiva di cui abbiamo trattato, non solo in senso strettamente clinico, hanno appunto una natura psicologico-morale, la loro dinamica credo venga a rappresentare il rovescio speculare di quelle a cui si richiama Honneth: al contrario di queste ultime, che innescano la dinamica delle lotte per il riconoscimento, esse conducono alla stasi, all'impotenza, all'implosione.

Il passaggio fondamentale che secondo Honneth permette il passaggio dal disrispetto subito individualmente alla nascita di lotte collettive volte a trasformare quelle condizioni che hanno determinato le ingiustizie in questione, la cui formazione permette nel contempo una reinterpretazione emancipatoria del disrispetto subito, ha come fondamentale precondizione un'operazione di traduzione: le esperienze debbono essere preliminarmente

17. Cfr. Honneth A., Lotta per il riconoscimento, cit., soprattutto pp. $160 \mathrm{sgg} ., 190 \mathrm{sgg}$.

18. Cfr. Fraser N., Honneth A., Redistribuzione o riconoscimento?, cit., soprattutto pp. 190 sgg. 
interpretate in termini di lesioni o privazione di diritti che, in quanto tali, valicano i confini del singolo individuo. Più da vicino, è necessario disporre di quella «semantica collettiva» che consente «di interpretare le esperienze personali di delusione come qualcosa che non concerne soltanto l'Io individuale, ma appunto una cerchia di molti altri soggetti»; in altri termini, deve essere «sufficientemente solido» il «ponte semantico» che permette «l'elaborazione di un'identità collettiva» e che, dischiudendo la via all'«azione politica», «strappa» i soggetti «dalla situazione paralizzante di uno svilimento subito passivamente, aiutandoli, corrispondentemente, a realizzare un nuovo, positivo rapporto con se stessi» ${ }^{19}$. Rispetto alle reazioni di natura depressiva si potrebbe dire che la situazione di svilimento paralizzante resti tale, e anzi in certo qual modo si aggravi: i soggetti sembrano rivoltarsi direttamente contro loro stessi, auto-annichilendosi. Si tratta perciò di esperienze che proporrei di definire «regressive»: il disrispetto subito, anziché innescare una reazione emancipatoria, si rovescia contro il soggetto stesso. La rabbia, la carica di aggressività può cosi prendere come oggetto direttamente l'Io, e condurre quindi alla disperazione, all'implosione; oppure può essere proiettata all'esterno, verso meccanismi e figure impersonali e lontane, sì da indurre il soggetto a percepirsi nei termini di una vittima predestinata in balia degli eventi, del tutto impotente.

\section{V}

U na tale dinamica sembrerebbe indicare che il «ponte semantico», e certo anche i pilastri sociali sui quali venne eretto, atto a trasporre le esperienze individuali di ingiustizia sul più ampio piano socio-politico, sia ormai pericolante. Allorché ci si muove in questa dimensione ridiviene però cruciale, tra una molteplicità di concause eterogenee, contemplare il ruolo giocato dall'affermazione del modello neoliberista; ritorniamo così al meccanismo della flessibilità e al nuovo quadro culturale. La flessibilizzazione del mercato (e delle forme) del lavoro ha difatti senza dubbio contributo in modo determinante a spezzare quelle fondamentali dinamiche della solidarietà, frammentando e atomizzando il mondo del lavoro ${ }^{20}$, che rappresentano una delle precondizioni di base per l'elaborazione sia della semantica sia delle identità collettive neces-

19. Cfr. Honneth A., Lotta per il riconoscimento, cit., pp. 191-193.

20. Sui processi correlati di «de-collettivizzazione» e «re-individualizzazione» vedi Castel R., L'insécurité sociale. Qu'est-ce qu'être protégé?, Paris 2003, trad. it. L'insicurezza sociale. Che significa essere protetti?, Einaudi, Torino 2004, pp. 40-47. 
sarie alla costruzione di movimenti di lotta. Sincronicamente, la dinamica paradossale attraverso cui ha operato la nuova configurazione ideologica è stata tale per cui il peso del sempre più diffuso malessere sociale è ricaduto sulle spalle dei singoli in termini di responsabilità personale. Carico, o meglio sovraccarico che sembrerebbe aver contributo alla diffusione di reazioni di natura depressiva.

Se è così, la possibilità di riattivare i potenziali emancipativi normativi inespressi nelle esperienze di ingiustizia sociale e di favorire sincronicamente il dirottamento delle cariche emotive e pulsionali in gioco, sì da volgerle da una direzione per così dire regressiva a una progressiva, si traduce allora nella necessità preliminare di ricostruire il ponte crollato. Nel momento stesso in cui tale operazione rappresenta il tentativo di contrapporsi alla pressione della nuova configurazione ideologica, a sua volta saldamente supportata dagli apparati egemonici del nuovo modello, essa viene nel contempo a configurarsi come una lotta per l'egemonia, in una accezione prossima a quella adottata da Gramsci quando scriveva che una «comprensione critica di se stessi» in grado di superare la condizione «in cui la contraddittorietà della coscienza non permette nessuna azione, nessuna decisione, nessuna scelta e produce uno stato di passività morale e politica», transita «attraverso una lotta di "egemonie" politiche, di direzioni contrastanti, prima nel campo dell'etica, poi della politica» ${ }^{21}$. È questa, credo, una delle vie obbligate da percorrere se si vuole spezzare il circolo perverso attraverso cui il peggioramento delle condizioni di vita contribuisce ad aggravare ed esasperare una forma di sofferenza psichica che, paralizzando i soggetti oggetto di ingiustizie sociali, prosciuga alla base le risorse motivazionali necessarie per costruire quei movimenti collettivi in grado di contrapporsi a questa stessa dinamica e, quindi, al suo impatto depressivo, svuotante.

\section{Sintesi}

Il saggio mira a individuare e delucidare alcuni nessi causali tra il concomitante incremento dei fenomeni depressivi, non solo in senso strettamente clinico, e l'affermazione del nuovo modello capitalistico avvenuti nei paesi occidentali dai primi anni settanta ad oggi. Oltre che sul meccanismo della flessibilità del mercato del lavoro, si insiste in particolare sulle dinamiche para-

21. Gramsci A., Quaderni del carcere, Quaderno 11 (XVIII) p. 16bis; nello stesso senso vedi Q. 13 (XXX), § 14-17. 
dossali delle istanze etiche e morali della nuova configurazione ideologica. Ricorrendo anche alla categoria di egemonia, vengono da ultimo approntati degli strumenti teorici finalizzati a riattivare i potenziali emancipativi frustrati nella sofferenza sociale di natura depressiva e regressiva.

Parole chiave: capitalismo, filosofia sociale, depressione, egemonia.

\section{Summary}

The paper aims to single out and clarify some causal connections between the concomitant growth of depressive phenomena, not only in the strict clinical sense, and the establishment of the new capitalist model, which has taken place in Western countries from the early seventies until today. As well as on the mechanism of labour market flexibility, the essay dwells in particular on the paradoxical dynamics of the ethical and moral ideals of the new ideological configuration. Finally, the paper will also use the category of hegemony to offer some theoretical instruments directed at reactivating the emancipatory potentials frustrated by social suffering of a depressive and regressive nature.

Keywords: capitalism, social philosophy, depression, hegemony. 